\title{
Finger volume pulse waveforms facilitate reliable assessment of heart rate variability, but not blood pressure variability or baroreflex function
}

\author{
Jonathan R Linder ${ }^{3,7}$, Harald M Stauss ${ }^{5}$, Holly Gindes ${ }^{3}$, Gary L Pierce ${ }^{5}$, Nicholas H Von Bergen², William G Haynes ${ }^{1,4}$
} and Jess G Fiedorowicz, ${ }^{1,3,6,8^{*}}$

\begin{abstract}
Background: We sought to determine whether heart rate variability (HRV), blood pressure (BP) variability, and baroreceptor-heart rate reflex sensitivity can be reliably assessed using finger volume pulse waveforms obtained from the commercially available EndoPAT device.

Methods: Non-invasive BP (Finometer Pro as a non-invasive standard) and finger volume (EndoPAT) waveforms were recorded in 65 adults (37 \pm 14 years; $60 \%$ female) and systolic BP and heart rate (HR) time series were derived after calibrating the EndoPAT signal based on systolic and diastolic BP values obtained by a sphygomomanometer. Transfer function analyses were performed to test for coherence between systolic BP and HR time series derived from the Finometer and EndoPAT devices. Time-domain HRV parameters, frequency domain HR and systolic BP variability parameters, and baroreflex sensitivity (sequence technique) were computed from Finometer- and EndoPAT-derived time series and intraclass correlation coefficients (ICC) were calculated.

Results: Squared coherence between systolic BP time series derived from the Finometer and EndoPAT devices was low, suggesting poor correlation. In contrast, squared coherence between HR time series derived from the two devices was excellent [High Frequency $(H F)=0.80$, Low Frequency $(L F)=0.81$, with gain values close to 1.0. ICC values for time- and frequency-domain HRV parameters were excellent ( $>0.9$ except for relative HF HRV, which was 0.77), while ICC values for frequency-domain BP variability parameters and baroreceptor-HR reflex sensitivity were low.

Conclusions: Finger volume pulse waveforms can be used to reliably assess both time-domain and frequency-domain HR variability. However, frequency domain BP variability parameters cannot be reliably assessed from finger volume pulse waveforms using the simple calibration technique used in this study.
\end{abstract}

Keywords: EndoPAT, Finometer Pro, Cardiovascular function, Device validation

\section{Background}

Autonomic regulation of heart rate (HR) and blood pressure (BP) is commonly cited as an indicator of cardiovascular health in humans [1-4]. Time- and frequency-domain HR variability (HRV) provide reliable estimates of both sympathetic and parasympathetic modulation of cardiac function $[2,3,5,6]$, while low frequency BP variability (BPV) reflects sympathetic modulation of vascular tone $[4,7,8]$.

\footnotetext{
* Correspondence: jess-fiedorowicz@uiowa.edu

'Department of Internal Medicine, The University of lowa, lowa City, IA 52242, USA

${ }^{3}$ Department of Psychiatry, Roy J. and Lucille A. Carver College of Medicine, The University of lowa, lowa City, IA 52242, USA

Full list of author information is available at the end of the article
}

Baroreceptor-HR reflex sensitivity is another common measure of cardio-autonomic health, providing insight into cardiovascular regulation by the baroreceptors $[9,10]$.

Time-domain HRV can be reliably assessed by deriving the standard deviation of $\mathrm{NN}$ intervals (SDNN) and the root-mean-square of successive differences (RMSSD) from normal sinus rhythm pulse intervals $(\mathrm{NN})$. SDNN is the standard deviation of all $\mathrm{NN}$ intervals over a fixed amount of time, usually 5 -minutes $[1,3]$. NN intervals are the measured intervals between consecutive heartbeats originating from a sinus rhythm. SDNN is an indicator of both sympathetic and parasympathetic cardiac inputs, and is thus a 
representative estimate of overall autonomic regulation of HRV. RMSSD is the square root of the mean of the squared differences between successive NN intervals. RMSSD is considered to reflect mainly parasympathetic and less sympathetic modulation of cardiac function [1].

Spectral analysis can be used to decompose oscillatory components of HRV and BPV into high frequency (HF), low frequency (LF), and very low frequency (VLF) bands. For HRV, the HF range (0.15-0.4) is representative of respiration-linked parasympathetic cardiac modulation, LF (0.06-0.15) is considered to be indicative of both parasympathetic and sympathetic modulation, and VLF (0.02-0.06) is sometimes considered representative of sympathetic cardiac modulation, but its true physiologic/pathophysiologic role is unclear $[1,2,7]$. For BPV in humans, the HF (0.15-0.4) component is largely dependent on respiration-linked fluctuations in stroke volume possibly caused by respiratory mechanics. LF BPV (0.0750.15 ) is mostly representative of sympathetic modulation of vascular tone, while VLF BPV (0.02-0.07) results from several mechanisms, including myogenic vascular control and endocrine factors (e.g., renin-angiotensin system) $[4,8,11]$.

In this study, we sought to validate the measurements obtained from a commercially available EndoPAT device (Itamar Medical Ltd., Caesarea, Israel) using finger volume pulse waveforms to derive HRV and BPV parameters and to estimate baroreceptor-HR reflex sensitivity. BP waveforms obtained from a commercially available Finometer Pro device (Finapres Medical Systems, Amsterdam, The Netherlands) were used to validate the EndoPATderived HRV and BPV parameters and baroreceptor-HR reflex sensitivity. The EndoPAT device measures arterial pulsatile volume changes in the fingertips by plethysmography. This reportedly allows assessment of arterial dilator capability, suggested to be an indicator of overall cardiovascular health [12]. This technology has the potential to allow non-invasive measurement of HRV and BPV, however has yet to be validated for these uses. The EndoPAT device provides waveforms that potentially allow for reliable beat-by-beat $\mathrm{HR}$ and $\mathrm{BP}$ trends to be derived and subsequently used for HRV, BPV, and baroreflex analyses. The Finometer Pro, a non-invasive BP monitoring system, evaluates finger arterial BP measurements detected by an infrared photoplethysmograph and a finger pressure cuff. The Finometer Pro can be used as a non-invasive standard for measuring HRV and BPV in humans $[13,14]$. The Finometer Pro and related machines have been validated in a wide variety of patient populations for HRV, BPV and baroreflex analyses [15-17].

We hypothesized that the EndoPAT finger volume pulse waveforms would provide reliable estimates for both time-domain and frequency-domain HRV. We also sought to determine whether these waveforms can be used to estimate BPV and/or baroreflex sensitivity after appropriate calibration.

\section{Methods \\ Sample}

The protocol was approved by the Institutional Review Board at the University of Iowa and was conducted in compliance with the Declaration of Helsinki. Participants were recruited using a University-wide email and a local print advertisement at the University of Iowa Hospitals and Clinics. Participants were excluded if they had significant hand injuries. All participants were asked to abstain from food, caffeine, and tobacco for two hours before each visit. We obtained informed consent from each participant, and a member of the research team assessed history of illness, recreational drug exposure, alcohol exposure, and tobacco exposure (pack per day"years). Alcohol exposure was assessed using the AUDIT$C$ screener [18], and participants were classified as either positive or negative for alcohol misuse. Men were classified under alcohol misuse following an AUDIT-C result of 4 or higher, while women were classified under alcohol misuse following an AUDIT-C result of 3 or higher.

\section{HRV, BPV, and baroreflex assessment}

For all participants, BP waveforms were recorded noninvasively in the middle finger of the left hand for 30 minutes at a sampling rate of $200 \mathrm{~Hz}$ using the Finometer Pro device (non-invasive "gold standard"). Simultaneously, finger volume pulse waveforms were also recorded in the index fingers of both hands noninvasively using the EndoPAT device, sampling at a frequency of $128 \mathrm{~Hz}$. Only the EndoPAT recordings for the left hand were used for subsequent analyses. The non-calibrated finger volume pulse waveforms were calibrated so that the average systolic peaks and diastolic troughs of the 30-minute waveforms corresponded to the systolic and diastolic BP values obtained using a sphygmomanometer at the beginning of the protocol. From these waveforms, systolic BP and heart rate were derived using the Analyzer module of the freely available HemoLab software (http://www.haraldstauss.com/ HaraldStaussScientific/hemolab/default.html) on a beatby-beat basis. HR and systolic BP were derived from the calibrated EndoPAT waveform. From this signal, beat-by-beat trends in heart rate and systolic BP were derived in the HemoLab software analyzer module. The Finometer provided an automatically derived trend for both HR and BP. No filters were used during data acquisition or subsequent data analysis.

Heart rate and systolic BP values affected by recording artifacts or cardiac arrhythmias were visually detected and replaced by interpolated values. The following analysis was performed using the Batch Processor module 
of the HemoLab software: Time-domain HRV parameters were calculated from the beat-by-beat HR time series using a stationary artifact free segment of 5-minute duration. For frequency-domain analysis, the beat-by-beat systolic BP and HR time series were interpolated (cubic spline) to obtain equidistant time series at $10 \mathrm{~Hz}$ sampling rate. Absolute and relative (absolute spectral power divided by total spectral power) spectral powers were calculated in the VLF $(0.02-0.05 \mathrm{~Hz})$ [2,4], LF (0.05$0.15 \mathrm{~Hz})$, and $\mathrm{HF}(0.15-0.50 \mathrm{~Hz})$ bands by applying the fast Fourier transform (FFT, 410 s long segments, 50\% overlap) to the full 30 minute long time series. Timedomain analysis was limited to 5-minute segments, as recommended [1], because duration affects the size of the results in time-domain analysis. Results from frequencydomain analysis, on the other hand, are not affected by duration of the segments analyzed.

Baroreceptor-heart rate reflex sensitivity was calculated from the 30 minute Finometer-derived BP and EndoPAT-derived calibrated finger volume pulse waveforms using the sequence technique implemented in the Analyzer module of the HemoLab software. The entire recording was selected for analysis of each device, separately. Systolic BP was analyzed using the "CalculateBaroreflex" function with an " $\mathrm{R}$ for inclusion" of 0.8. A delay of three heartbeats was selected between the beatby-beat systolic BP and pulse interval values.

\section{Transfer function analysis}

In order to assess the correlation between Finometerderived and EndoPAT-derived time series, transfer function analysis was performed that provides the squared coherence and the gain between the two time series. The squared coherence provides values (between 0 and 1 ) similar to the $R^{2}$ value of a correlation analysis for each frequency component of the two time series. The gain of the transfer function can be seen as a factor by which each frequency component of one time series is amplified (gain $>1.0$ ) or reduced (gain <1.0) compared to the other time series. Transfer function analysis was performed from the spline interpolated $(10 \mathrm{~Hz})$ systolic BP time series derived from the EndoPAT- and Finometer-derived waveforms. The same protocol was performed for the HR time series derived from both respective machines. The squared coherence function $\gamma(\mathrm{q})$ and gain $\mathrm{H}(\mathrm{q})$ of the transfer functions were calculated on the basis of the autospectral density functions and the cross-spectrum of the input (Finometer-derived time series) and output (EndoPATderived) functions. This was done using the Batch processor module of the HemoLab software.

Subsequently, the maximum gain and coherence values for both the HF and LF spectral domains were calculated for each participant. This was done for the transfer functions calculated from both the HR and BP time series.
Due to systematic detection of cardiac arrhythmias, four participants were excluded from the transfer function analysis.

\section{Statistical analyses}

Data are presented as mean \pm SD. Study data were collected and managed using REDCap (Research Electronic Data Capture) tools hosted at The University of Iowa Hospitals and Clinics. Data was merged using SAS 9.3 (SAS Institute Inc., Cary, NC) and descriptive statistics for the sample were compiled. Intraclass correlation coefficients between parameters derived from Finometer-(BP) and EndoPAT-derived (finger volume pulse) waveforms were calculated using SPSS Statistics 19.0 (IBM Corp. Armonk, NY). Bland-Altman plots were created using SigmaPlot 12.0 (Systat Software, San Jose, CA) to display agreement across the range of observed values.

\section{Results}

Sociodemographic and clinical characteristics of our sample are outlined in Table 1 . The sample had a mean (SD) age of 37 (14) and was $60 \%$ female.

Transfer function analysis (Table 2) revealed that squared coherence for systolic BP time series derived from the Finometer and EndoPAT devices was relatively low $(<0.35)$ for the LF and HF bands. This finding indicates

Table 1 Sociodemographic and clinical characteristics $(\mathrm{N}=65)$

\begin{tabular}{lc}
\hline & Mean (SD) \\
\hline Age & $37(14)$ \\
Pack years (Smoking) & \\
$\quad$ Entire sample & $2.6(7.6)$ \\
$\quad$ Smokers only & $12.4(12.5)$ \\
Body mass index (kg/m2) & $27.9(6.5)$ \\
Heart rate (bpm) & $70.9(13)$ \\
Systolic blood pressure (mmHg) & $119.5(13.0)$ \\
Diastolic blood pressure (mmHg) & $74.9(8.6)$ \\
Respiratory rate (min $\left.{ }^{-1}\right)$ & $13.4(3)$ \\
& $\mathrm{N}(\%)$ \\
Female gender & $39(60 \%)$ \\
White, not hispanic & $61(93.8 \%)$ \\
Unmarried & $32(48 \%)$ \\
Unemployed & $3(4.6 \%)$ \\
Alcohol misuse & $31(47 \%)$ \\
History of tobacco use & $14(22 \%)$ \\
Heart attack & $1(1.5 \%)$ \\
High blood pressure & $9(13.6 \%)$ \\
Diabetes or high blood sugar & $4(6 \%)$ \\
\hline
\end{tabular}


Table 2 Transfer function analysis between finometerand EndoPAT-derived time series

\begin{tabular}{lccccc}
\hline & \multicolumn{2}{c}{ Low frequency } & & \multicolumn{2}{c}{ High frequency } \\
\cline { 2 - 3 } Mean(SD) & Coherence & Gain & & Coherence & Gain \\
\hline Systolic BP & $0.33(0.13)$ & $0.90(0.45)$ & & $0.27(0.08)$ & $0.55(0.34)$ \\
HR & $0.81(0.17)$ & $0.94(0.14)$ & & $0.80(0.17)$ & $1.03(0.22)$ \\
\hline
\end{tabular}

a poor correlation between systolic BP values derived from the Finometer and EndoPAT devices. As a result of the low squared coherence, we don't feel that the gain values can be interpreted in a meaningful way.

In contrast to systolic BP, the squared coherence for the HR time series derived from the two devices was high $(\mathrm{HF}=0.80, \mathrm{LF}=0.81)$, indicating excellent agreement between Finometer- and EndoPAT- derived HR time series. In addition, the gain $\sim 1.0$ indicates that the HR values correspond in absolute terms.

Results of our primary analysis are summarized in Table 3. Intraclass correlation coefficients (ICC) for timedomain HRV showed a strong association for both SDNN (0.99) and RMSSD (0.96). Frequency-domain HRV showed similarly strong ICCs for both absolute and relative measures of VLF, LF, and HF spectral powers (ranging from 0.77 to 0.97 , Table 3). The frequency domain BPV analysis garnered much weaker ICCs than HRV analysis with ICC values ranging from 0.34 to 0.64 (Table 3 ).

Baroreceptor-heart rate reflex sensitivity values derived from the two waveforms were not well correlated (ICC $=$ 0.27). From the EndoPAT-derived time series, only 4.5 (SD 4.0) baroreflex sequences were detected per 1000 heartbeats compared to $6.1(8.0)$ sequences per 1000 heart beats detected in the Finometer-derived time series. However, this difference in number of sequences was not significant (Wilcoxon Signed Rank $\mathrm{S}=-211, \mathrm{p}=0.16$ ). EndoPAT-derived time series tended to overestimate baroreflex gain relative to the Finometer-derived time series [mean (SD)] 23.9 (12.0) ms/mmHg vs. 15.7 (8.2) ms/mmHg and EndoPAT-derived baroreflex gains did not appear to reliably correlate to the Finometer-derived gains (ICC $=0.27,95 \%$ C.I. 0.02-0.49).

Comparison by Bland-Altman plots supported the results obtained by transfer function analysis and intra-class correlation analysis, indicating that EndoPAT and Finometer provide similar results for both time - (Figure 1) and frequency-domain (Figure 2) HRV parameters, across the range of observed values. For all frequency domain BPV parameters (Figure 3), the Bland-Altman plots demonstrated poor agreement between Finometer- and EndoPATderived values.

\section{Discussion}

The aim of this study was to investigate if HR and systolic BP time series derived from finger volume pulse waveforms provided by the EndoPAT device allow for reliable estimation of $\mathrm{HRV}$ and BPV parameters. As a validated standard, $\mathrm{HR}$ and systolic $\mathrm{BP}$ time series obtained from the Finometer device were used. To investigate if the EndoPAT-derived $\mathrm{HR}$ and systolic BP time series correspond to the time series derived from the Finometer device, transfer function analysis was performed. The squared coherence of the transfer function revealed excellent correlation between the HR time series derived from the two devices but indicated poor correlation for the systolic BP time series. Even without looking at the actual HRV and BPV parameters, this finding already indicates that HRV parameters may be reliably derived from waveforms provided by the EndoPAT device, while it is highly questionable if systolic BPV can be reliably assessed from the finger volume pulse waveforms. To some degree, this result was expected, as the pulse-synchronous oscillatory pattern of the finger volume pulse waveform should allow for reasonably accurate determination of inter-beat intervals. However, the waveform of the finger volume pulse is distorted relative to the standard pressure waveform provided by the Finometer device and, thus, accurate determination of beat-by-beat systolic BP may not be possible using a simple calibration procedure based on sphygmomanometer-derived BP values.

Measures of SDNN and RMSSD, as well as VLF, LF, and HF spectral HRV derived from the EndoPAT waveforms, were all in strong agreement (high ICC values) with these parameters derived from the Finometer waveforms. The ICC value for relative HF spectral power of HRV (0.77) was not as high as for VLF and LF HRV (0.94 and 0.92) but still significant. While spectral parameters of BPV derived from the EndoPAT device did not correlate well with BPV parameters derived from the Finometer, the ICC value for relative LF BPV was in the acceptable range (0.64). However, given the low LF coherence between systolic BP time series derived from EndoPAT and Finometer, this ICC value of 0.64 should be considered cautiously and may not indicate that relative LF spectral power of systolic BP can be reliably derived from EndoPAT-derived waveforms.

The ICC value for baroreflex sensitivity was even less than the ICC value for BPV. The low ICC value we observed for the baroreflex gain suggests that time series derived from the EndoPAT device do not reliably assess baroreflex function. Baroreflex analysis based on EndoPATderived waveforms tended to overestimate baroreflex sensitivity, and only detected approximately two thirds of the baroreflex sequences detected by Finometerderived waveforms.

The current study is not without limitations. Both machines, to allow for simultaneous recordings, were applied to the same hand - whereas in a typical use, 
Table 3 Intraclass correlation coefficients for primary outcomes

\begin{tabular}{lccc}
\hline \multicolumn{1}{c}{ Measures } & \multicolumn{3}{c}{ Intraclass correlation coefficients between EndoPAT and finometer } \\
\cline { 2 - 4 } & & \multicolumn{2}{c}{ 95\% Confidence interval } \\
\cline { 2 - 4 } & ICC & Lower bound & Upper bound \\
\hline SDNN & 0.99 & 0.98 & 0.99 \\
Absolute VLF of HRV & 0.96 & 0.94 & 0.98 \\
Relative VLF of HRV & 0.94 & 0.90 & 0.96 \\
Absolute LF of HRV & 0.94 & 0.90 & 0.96 \\
Relative LF of HRV & 0.94 & 0.90 & 0.96 \\
Absolute HF of HRV & 0.92 & 0.87 & 0.95 \\
Relative HF of HRV & 0.97 & 0.95 & 0.98 \\
Absolute VLF of BPV & 0.77 & 0.65 & 0.86 \\
Relative VLF of BPV & 0.34 & 0.11 & 0.54 \\
Absolute LV of BPV & 0.37 & 0.14 & 0.56 \\
Relative LF of BPV & 0.43 & 0.20 & 0.61 \\
Absolute HF of BPV & 0.64 & 0.47 & 0.77 \\
Relative HF of BPV & 0.57 & 0.38 & 0.71 \\
Baroreflex gain & 0.51 & 0.31 & 0.67 \\
\hline
\end{tabular}

the equipment would be used alone on a given hand. However, this did not cause any discernible disruption in the signals. Pulses may differ across digits. Additionally, signals for both machines were not recorded in unison and were sampled at different frequencies. These design issues might contribute to discrepancies between recordings from the two machines, which if anything would bias the observed correlation to an underestimate. Thus, the correlation between measures can be assumed to be at least as strong as our study indicates. HRV from electrocardiography was not available for comparison, though HRV measures from both instruments were highly consistent with each other. The
EndoPAT device includes a high-pass filter, the frequency of which is not provided, which may impact variability measures on spectral analysis, especially in the VLF range. Although the age range of our adult cohort spanned a broad range and included a balance of male and female participants, they were young and predominantly Caucasian, therefore caution should be used when extrapolating our findings to other ethnicities. While time domain parameters for HRV are generally accepted to have high inter- and intra-visit reproducibility, spectral measurements have been shown to be less reproducible between visits [19], and may thus be less useful clinically.

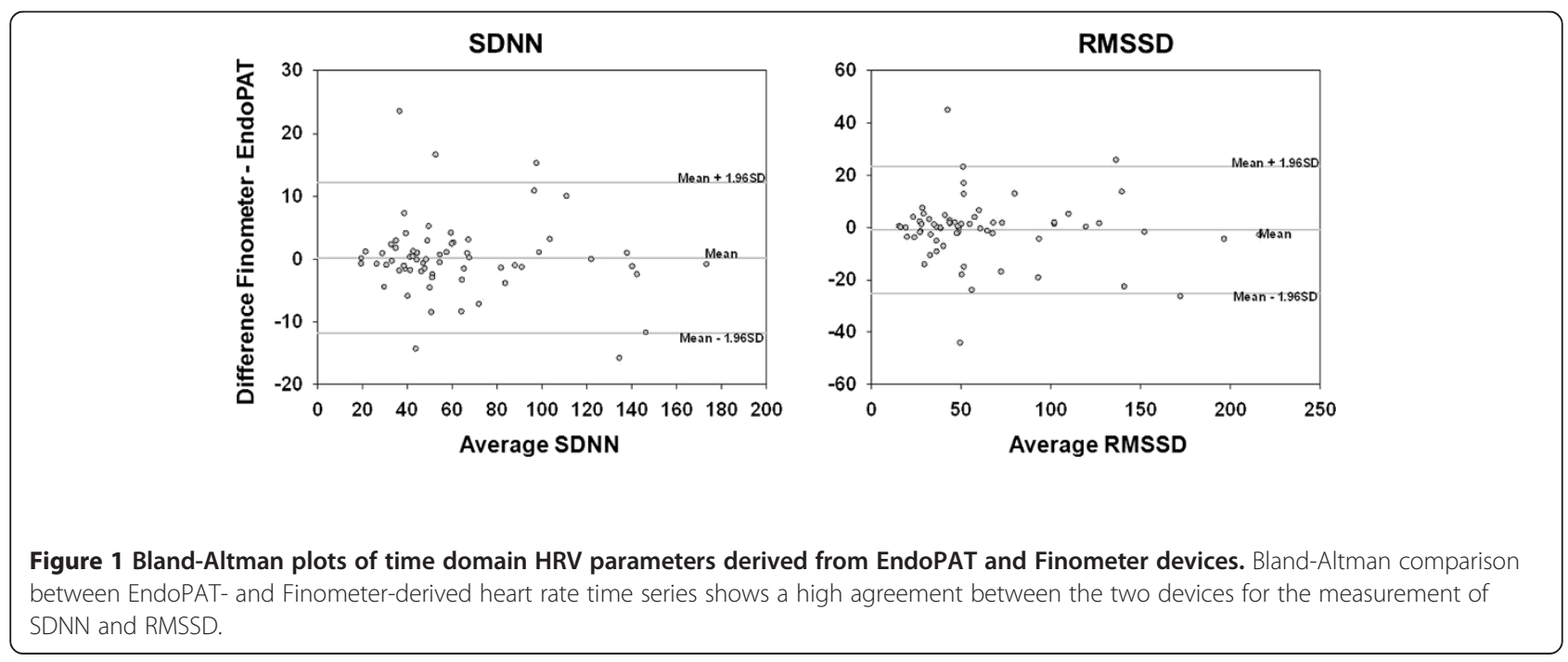




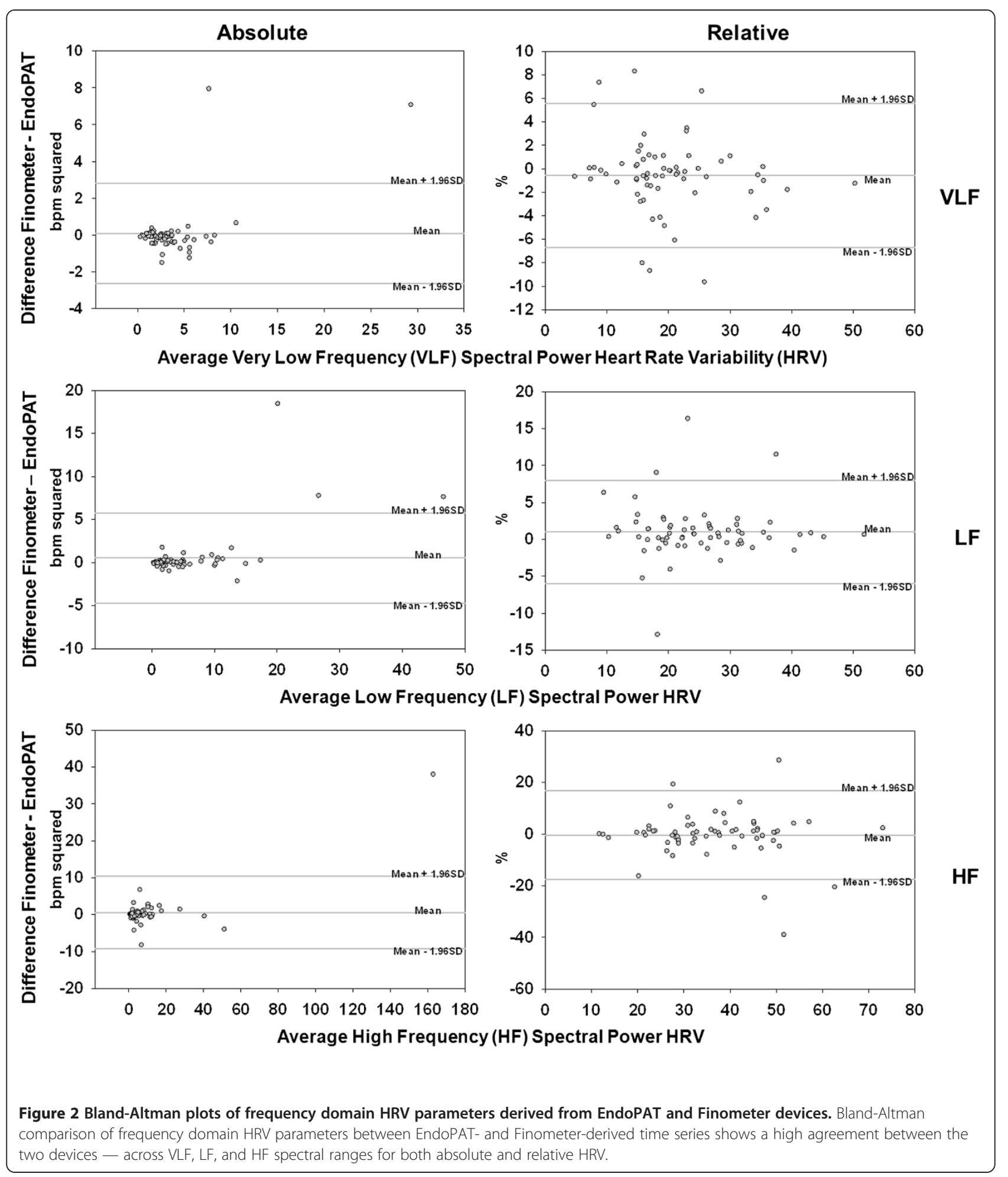

Even though the conclusion of this study is that parameters that depend on accurate BP waveforms, such as BPV or baroreflex function, cannot be reliably determined from the EndoPAT signal, one may speculate that it may be possible to design more advanced mathematical algorithms that may potentially be able to convert the finger volume pulse from the EndoPAT device into a BP waveform. E.g., a transfer function may be derived from the EndoPAT and Finometer recordings obtained in this study that may potentially allow for reconstruction of a more or less 


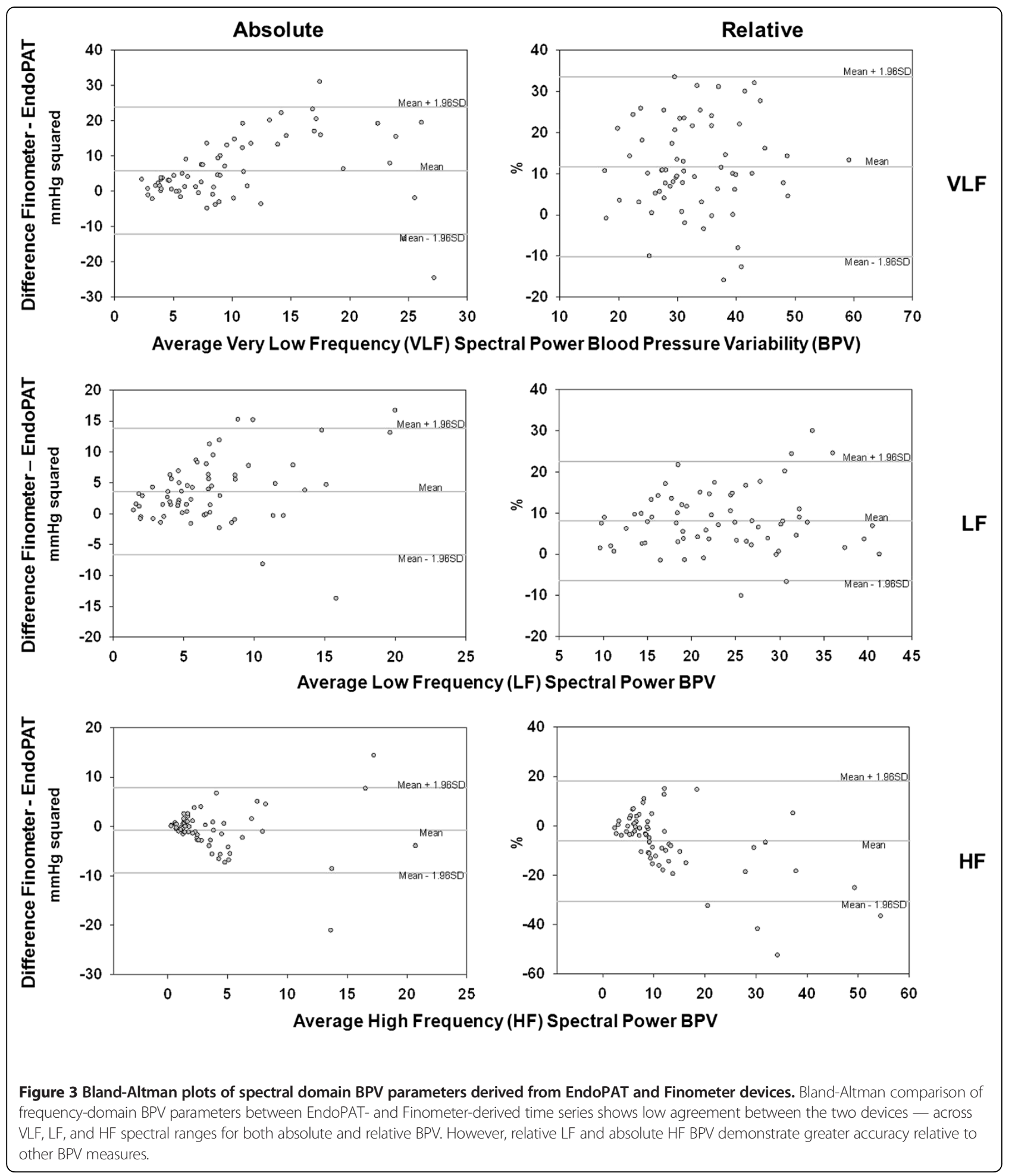

accurate BP waveform from the finger volume pulse signal provided by the EndoPAT device. However, such analysis is beyond the scope of this study. Similarly, our study is not applicable to the calculation of variability between routine BP readings in the clinic [20].

\section{Conclusions}

In summary, our findings support the hypothesis that HRV can be reliably assessed by analysis of the raw EndoPAT signal (i.e., finger volume pulse waveforms), which may allow researchers to assess cardiovascular/autonomic 
health in addition to the manufacturer's primary use which is to measure microvascular endothelial function. In contrast, the BP variability derived from the EndoPAT device showed low agreement with the measurements obtained from the Finometer, suggesting that the EndoPAT should not be used to assess BP variability. Still, while the EndoPAT may not be cost effective to use solely for HRV analysis, it may prove useful to employ the device for measuring HRV in subjects on which the device has already been used to assess endothelial function (i.e., retrospective analysis of existing recordings).

\section{Abbreviations}

BP: Blood pressure; BPV: Blood pressure variability; FFT: Fast fourier transform; HF: High frequency; HRV: Heart rate variability; ICC: Intraclass correlation coefficient; LF: Low frequency; NN: Normal sinus rhythm pulse intervals; RMSSD: Root-mean-square of successive differences; SDNN: Standard eviation of NN intervals; VLF: Very low frequency.

\section{Competing interests}

The authors declare that they have no competing interests.

\section{Authors' contributions}

JRL, NHV, WGH, and JGF designed the study. NHV provided the Finometer and JGF provided the EndoPAT. JGF funded the study, which was conducted in his lab. JRL and HG recruited participants and collected the data. JRL performed the analyses of HRV and BPV with assistance from HMS. JGF performed the statistical analysis. JRL, HMS, GLP, NHV, WGH, JGF were involved in interpreting the analyses. JRL and JGF drafted the manuscript, which was edited with co-authors. All authors read and approved the final manuscript.

\section{Acknowledgements}

Dr. Fiedorowicz is supported by the National Institutes of Health (K23MH083695, P01HL014388). William G. Haynes is currently employed by the Novartis Institute for Biomedical Research, Inc., Cambridge Massachusetts and was faculty at the University of lowa at the time the study was conducted.

\section{Author details}

'Department of Internal Medicine, The University of lowa, lowa City, IA 52242, USA. ${ }^{2}$ Department of Pediatrics, The University of lowa, lowa City, IA 52242, USA. ${ }^{3}$ Department of Psychiatry, Roy J. and Lucille A. Carver College of Medicine, The University of lowa, lowa City, IA 52242, USA. ${ }^{4}$ Institute for Clinical and Translational Science, The University of lowa, lowa City, IA 52242, USA. ${ }^{5}$ Department of Health and Human Physiology, College of Liberal Arts \& Sciences, The University of lowa, lowa City, IA 52242, USA. 'Department of Epidemiology, College of Public Health, The University of lowa, lowa City, IA 52242, USA. ${ }^{7}$ College of Pharmacy, The University of lowa, lowa City, IA 52242, USA. ${ }^{8} 200$ Hawkins Drive W278GH, lowa City, IA 52242-1057, USA.

Received: 27 June 2014 Accepted: 26 November 2014

Published: 9 December 2014

\section{References}

1. Malik M, Bigger JT, Camm AJ, Kleiger RE, Malliani A, Moss AJ, Schwartz PJ: Heart rate variability: Standards of measurement, physiological interpretation, and clinical use. Task Force of the European Society of Cardiology and the North American Society of Pacing and Electrophysiology. Eur Heart J 1996, 17(3):354-381

2. Cavalcanti S, Severi S, Chiari L, Avanzolini G, Enzmann G, Bianco G, Panzetta G Autonomic nervous function during haemodialysis assessed by spectral analysis of heart-rate variability. Clin Sci (Lond) 1997, 92(4):351-359.

3. Ewing DJ, Borsey DQ, Bellavere F, Clarke BF: Cardiac autonomic neuropathy in diabetes: comparison of measures of R-R interval variation. Diabetologia 1981, 21(1):18-24.
4. Stauss HM: Identification of blood pressure control mechanisms by power spectral analysis. Clin Exp Pharmacol Physiol 2007, 34(4):362-368.

5. Pagani M, Lombardi F, Guzzetti S, Rimoldi O, Furlan R, Pizzinelli P, Sandrone G, Malfatto G, Dell'Orto S, Piccaluga E, Turiel M, Baselli G, Cerutti S, Malliani A: Power spectral analysis of heart rate and arterial pressure variabilities as a marker of sympatho-vagal interaction in man and conscious dog. Circ Res 1986, 59(2):178-193.

6. Sandercock GR, Bromley P, Brodie DA: Reliability of three commercially available heart rate variability instruments using short-term (5-min) recordings. Clin Physiol Funct Imaging 2004, 24(6):359-367.

7. Latalova K, Prasko J, Diveky T, Grambal A, Kamaradova D, Velartova H, Salinger J, Opavsky J: Autonomic nervous system in euthymic patients with bipolar affective disorder. Neuro Endocrinol Lett 2010, 31(6):829-836.

8. Parati G, Saul JP, Di Rienzo M, Mancia G: Spectral analysis of blood pressure and heart rate variability in evaluating cardiovascular regulation. A critical appraisal. Hypertens 1995, 25(6):1276-1286.

9. Parati G: Arterial baroreflex control of heart rate: determining factors and methods to assess its spontaneous modulation. J Physiol 2005, 565(Pt 3):706-707.

10. Parati G, Di Rienzo M, Mancia G: How to measure baroreflex sensitivity: from the cardiovascular laboratory to daily life. J Hypertens 2000, 18(1):7-19.

11. Ponchon $\mathrm{P}$, Elghozi JL: Contribution of the renin-angiotensin and kallikrein-kinin systems to short-term variability of blood pressure in two-kidney, one-clip hypertensive rats. Eur J Pharmacol 1996, 297(1-2):61-70.

12. Hamburg NM, Keyes MJ, Larson MG, Vasan RS, Schnabel R, Pryde MM, Mitchell GF, Sheffy J, Vita JA, Benjamin EJ: Cross-sectional relations of digital vascular function to cardiovascular risk factors in the Framingham Heart Study. Circulation 2008, 117(19):2467-2474.

13. Chin KY, Panerai RB: Comparative study of Finapres devices. Blood Press Monit 2012, 17(4):171-178

14. Pinna GD, Maestri R: New criteria for estimating baroreflex sensitivity using the transfer function method. Med Biol Eng Comput 2002, 40(1):79-84

15. Guelen I, Westerhof BE, Van Der Sar GL, Van Montfrans GA, Kiemeneij F, Wesseling KH, Bos WJ: Finometer, finger pressure measurements with the possibility to reconstruct brachial pressure. Blood Press Monit 2003, 8(1):27-30.

16. Guelen I, Westerhof BE, van der Sar GL, van Montfrans GA, Kiemeneij $F$ Wesseling KH, Bos WJ: Validation of brachial artery pressure reconstruction from finger arterial pressure. J Hypertens 2008, 26(7):1321-1327.

17. Schutte $A E$, Huisman HW, van Rooyen JM, Malan NT, Schutte R: Validation of the Finometer device for measurement of blood pressure in black women. J Hum Hypertens 2004, 18(2):79-84.

18. Bush K, Kivlahan DR, McDonell MB, Fihn SD, Bradley KA: The AUDIT alcohol consumption questions (AUDIT-C): an effective brief screening test for problem drinking. Ambulatory Care Quality Improvement Project (ACQUIP). Alcohol Use Disorders Identification Test. Arch Intern Med 1998, 158(16):1789-1795

19. La Fountaine MF, Wecht JM, Spungen AM, Bauman WA: Intra-inter visit reproducibility of short-term linear and nonlinear measurement of heart rate variability in tetraplegia and neurologically intact controls. Physiol Meas 2010, 31(3):363-374.

20. Rothwell PM, Howard SC, Dolan E, O'Brien E, Dobson JE, Dahlof B, Sever PS, Poulter NR: Prognostic significance of visit-to-visit variability, maximum systolic blood pressure, and episodic hypertension. Lancet 2010, 375(9718):895-905.

doi:10.1186/1471-2261-14-180

Cite this article as: Linder et al:: Finger volume pulse waveforms facilitate reliable assessment of heart rate variability, but not blood pressure variability or baroreflex function. BMC Cardiovascular Disorders $201414: 180$ 